\title{
Ueber Chrysomeliden und Coccinelliden. aus Rhodesia
}

$$
\text { Von }
$$

J. We is e.

In den letzten Jahren sammelte Herr A. Bodong, der Vertreter mehrerer deutscher Firmen, mit grofsem Geschick in der Umgebung von Umtali in Britisch Central-Afrika und sandte seine Ausbeute zur Bestimmung gröfstenteils an Hrn. Péringuey in Capstadt, welcher davon bereits eine kurze Liste veröffentlichte. Es machte mir nun viel Freude, im vorigen Sommer, als Hr. Bodong einige Zeit in Berlin weilte, die Chrysomeliden und Coccinelliden seiner Sammlung durchsehen zu können, denn ich lernte dadurch nicht nur eine Reihe hübscher Arten kennen, sondern auch eine sehr interessante Sammelgegend, in der zahlreiche Arten aus dem südlichen DeutschOstafrika, Natal, vom Caplande und aus Westafrika zusammentreffen. Hier sollen aufser den neuen auch die seltensten anderen Arten aufgezählt werden.

Bradylema ${ }^{1}$ ) robusta Lac. Mon. 312 wurde nach einem 우 aus Caffraria beschrieben, welches sich im hiesigen $\mathrm{kgl}$. Museum f. Naturk. befindet. Mit ihr ist höchst wahrscheinlich identisch die L. pallida Clark, Cat. App. 21 ( 1 ㅇ Zululand; Stevens), noch sicherer L. rufoadumbrata Clark p. 22 , die auf ein frisches, mifsfarbenes oder schlecht behandeltes von derselben Lokalität gegründet sein dürfte, dessen Fld. etwas zusammengeprefst worden sind. Clarks spärliche Angaben enthalten viele Flüchtigkeitsfehler, z. B. p. 21 "rufo-parallela", "genubus nigris" ${ }^{2}$ ); letztere Bezeichnung kehrt auch p. 22 unter rufoodumbrata wieder, wo sich auch die widersinnige Angabe findet, diese Art soll zır zweiten Gruppe Lacordaire's gehören und hinter crassipes gestellt werden. Ein drittes 오, welches mit den beiden vorhergenannten zusammen gefangen wưfde, betrachtete meiner Meinung nach Clark p. 69 als

1) $\mathrm{Zu}$ dieser Gruppe gehört auch noch grandis Klug, Lac. 309.

$\left.{ }^{2}\right)$ Es sind nicht die Knie schwarz, sondern nur die äufserste Basis der Schienen. Lacordaire bezeichnet diese Färbung genau mit: libiarum macula balilari nigra und "une petite tache tout-à-fait à la base des jambes noire".

Deutsche Entomol. Zeitschrift 1905. Heft II. 
Crioceris constricticollis! Endlich gehört nach der Beschreibung noch Lema transvaalensis Jac., Ann. Belg. 1893, 264, hierher, und die Art würde, die Richtigkeit meiner Annahme vorausgesetzt, folgendes Bild bieten:

Bradylema robusta Lac. 1845.

pallida Clark 1866.

rufoadumbrata Clark 1866.

constricticollis Clark 1866.

transvaalensis Jac. 1893.

Ich besitze die Art aus Britisch Ostafrika (Staudinger), Usambara (Hohenfriedeberg; Dr. Schulz) und von Umtali (Bodong). Der Körper ist strobgelb (Spiritus-Exemplare), hell bräunlich gelb oder rötlich gelbbraun, Glied 2-11 der Fühler, ein kleiner Fleck an der Basis der Schienen und die Tarsen schwarz. Die Spitze der Schienen ist àn der Innenseite oft angedunkelt, die Basis der einzelnen Tarsenglieder zuweilen rötlich, auch kann jeder der vier ersten Bauchringe an der Seite eine schwarze Makel besitzen und die Mitte der Ringe etwas angedunkelt sein. Die Stirnhöcker sind glatt, oben getrennt, unten in einen kurzen Längskiel vereint, der Raum zwischen den Stirnrinnen und Augen ist mehr oder weniger runzelig punktiert. Der Thorax ist nahe der Mitte stark verengt, hat statt der Querrinne nur einen leichten Eindruck und ein Grübchen in der Mitte vor dem Schildchen, ist über den Vorderecken zerstreut-, in der Mitte in 3-4 nicht regelmäfsigen Reiben punktiert, von denen sich hinten ein Streifen mit Punkten ror dem Quereindrucke bis in die Nähe der Seitengrube zieht. Die Punkte des Thorax sind fein, zuweilen fast erloschen, die Seiten divergieren gleichmälsig von der Seitengrube bis zur vorderen Borstenpore; Fld. in regelmäfsigen Reihen punktiert, die glatten oder fein gerunzelten $Z$ wischenstreifen mit einer Reihe feinerer Punkte.

Die Hinterschenkel des $\sigma^{\top}$ sind dicker wie die des $q$ und haben am Innenrande vor der Spitze einen grofsen Zahn, neben dem sich weiter nach aufsen ein kleiner Zahn orhebt. In die Vertiefung, die beide Zähne trennt, legt sich, wenn das Tier die Hinterbeine zusammenzieht, ein stumpfer Zahn, der sich unterhalb dęr Schienenspitze befindet. Von diesem Zahne an bis zar Spitze sind die Hinterschienen eine Spur breiter als an der Basis und an der Innenseite dicht behaart und mit einer Reihe zahnförmiger Höcker besetzt.

Crioceris Bohemani Baly und elongata Jac. Von letzterer wurde die Stammform gefangen, bei der die Flügeldecken 
pechbraun, an der Basis mehr oder weniger breit gelb gesäumt sind. In Jacoby's Abbildung, Genera Insectorum 23, t. 4, fig. 2, erscheinen wohl die Fld. zu hell, fast gleichmäfsig gelbbraun.

Sigrisma cylindrica Klug, Ws. D. Ent. Z. 1905, 54. Die schwarze, vorn verbreiterte Mittelbinde des Thorax ist zuweilen weit unterbrochen und auf eine mälsig grofse, hinten abgerundete Makel am Vorderrande und eine kurze, strichförmige vor dem Schildchen beschränkt. Diese helle Form bezeichne ich als ab. thoracica.

Poecilomorpha mashonan J Jac. (mashuana, Transact. 1895, 164, wohl ein Druckfehler!). Es lagen mir nur ơ mit einfarbig bräunlich gelben Flügeldccken vor. Die Art pafst in keine Gattung dieser Gruppe.

Protoclytra umtaliensis Jac.

Die Arten, für die ich den Namen Protoclytra vorschlage, haben in Ost- und Südafrika ihre Heimat; sie wurden als Camptolenes, Lachnaea und Titubaea beschrieben, woraus schon hervorgeht, dafs sie sich in keine der genannten Gattungen genau einfügen lassen. Es sind oberseits mehr oder weniger matte, dicht und kräftig punktierte Arten, die vor der Spitze der Flgd. Reste von tiefen Punktstreifen und rippenförmigen $Z_{w}$ ischenstreifen zeigen. Der Thorax besitzt in oder etwas vor der Mitte jederseits einen mehr oder weniger tiefen Quereindruck, hinter dem die Fläche bis zur Basis bei den meisten Arten schwarz gefärbt ist. Die Flgd. sind gelblich, zusammen mit 10 schwarzen Makeln: 2, 2, 1, von denen die beiden ersten in der Regel zu einer gemeinschaftlichen Querbinde in $\frac{1}{3}$ Länge vereint sind, die aufsen winkelig bis auf die Schulterheule nach vorn verlängert ist. Die beiden folgenden sind meist $z u$ einer gemeinschaftlichen geraden Querbinde hinter der Mizte verbunden, die letzte befindet sich nahe der Spitze. Das $ぇ$ hat einen weit gröfseren $\mathbf{K} \circ \mathrm{pf}$ als das $q$ und sehr verlängerte Vorderbeine. Es gehören hierher:

Camptolenes abyssinica Lef., Revue et Mag 1877, 223 von Tigré, Lachnaea salaamensis Ws, Archiv f. Naturg. Juli 1902, 126, aus Deutsch Ostafrika, Tilubaea umlaliensis Jac. Proceed. 1904, 238, und wahrscheinlich auch Camptolenes pubifrons Jac. Proceed. 1900, 209, von Delagoa, Titubaea rugosa Jac. Transact. 1895, 166, aus Afr. mer., thoracica Jac. Ann. Mag. 1898, 347, ebenfalls von Afr. mer., und preloriae Jac. 1. c. von Pretoria. 
324 J. Weise: Ueber Chrysomeliden und Coccinelliden aus Rhodesia.

\section{Diapromorpha zebra Lac. (?).}

Der Autor hat diese Art sehr kurz nach einem Stücke beschrieben und nur mit der veränderlich gefärbten argentata $\mathrm{F}$. verglichen, von der ihm auch nur 2 Exempl. vorlagen. Ich glaube daher nicht fehl zu gehen, wenn ich die vorliegenden Tiere von Umtali auf zebra beziehe, obwohl sie nicht ganz in der Zeichnung der Oberseite mit Lacordaires Angaben übereinstimmen. Die Diagnose würde lauten:

D. zebra: Subtus subcoeruleo-nigra, argenteo-sericea, supra nigra, nitida, antennis articulis secundo et tertio rufescentibus, pro thorace limbo subapicali utrinque dilatato limboque laterali flavis, elytris minus dense punctatis, apice etiam nitidis et obsolete strigosis, fascia basali utrinque abbreviala, fascia submedia ad humeros usque extensa, maculisque duabus ante apicem flavis. - Long. 11-12 mill.

Es fehlt diesen Stücken also die kurze gelbe Mittellinie des Thorax, die von dem gelben und angeblich völlig glatten Spitzensaum ausgehen soll, ebenso haben die Flgd. nicht drei schwarze, zackige Querbinden. Der Körperumrifs ist dem der nabe verwandten haemorrhagica am ähnlichsten, nur etwas kürzer und breiter Der Kopf ist schwarz, äufserst dicht, fein punktiert, dicht, kurz weifslich behaart, der Scheitel etwas gewölbt, mit feiner, flacher Mittelrinne. Fühler schwarz, die Spitze des ersten Gliedes unterseits, sowie Glied 2 und 3 rötlich. Thorax schwarz, mit gelbem Saume an der Spitze, welcher in der Mitte durch einen weiten Bogen verengt ist und an den Seiten in einen schmaleren Saum übergeht; die ganze Oberfläche ist dicht mit äufserst zarten Pünktchen und weitläutig gestellten grölseren Punkten besetzt, welche nur an den Seiten des schwarz gefärbten Teiles allmählich stärker und dichter werden. Fld. feiner und weniger dicht wie bei haemorrhagica punktiert, jedoch an der Spitze glänzender, im ganzen ähnlich gezeichnet, aber die erste schwarze Querbinde erreicht vor der Schulter nicht ganz die Basis und die beiden gelben Flecke vor der Spitze sind ähnlich wie in den dunklen Formen von argentata gestellt. Prosternum zwischen den Hüften mälsig breit, hinten in einen überhängenden Fortsatz verlängert, der leicht ansteigt und ziemlich so hoch als das Mesosternum ist.

Ursprünglich hatte Lacordaire in seiner Monographie die Gattungen Diapromorpha und Peploptera, auf deren Unterschied ich hier hinweisen möchte, als Subgenera mit Clyıra verbunden, Chapuis, Genera 10, 121, nur die beiden letzten. Obwohl sich diese in den typischen Formen an dem flachen (Diapromorpha) oder tiefen 
Ausschnitte im Seitenrande der Fld. hinter der Schulter (Peploptera), dem wenig oder stark entwickelten Bauche, dem völlig bedeckten oder vorragenden Pygidium und den verworren oder gereiht punktierten Flgd. leicht unterscheiden lassen, genügen diese Merkmale bei den $Z$ wischenformen nicht, und man kommt in Zweifel, wohin eine Art wirklich zu stellen ist. Ich möchte deshalb noch als Unterschiede anführen: Die Fld. von Diapromorpha sind auf dem Abfalle zur Spitze stets mit Längsrissen bedeckt, deren $\mathrm{Z}$ wischenräume feine Längsrunzeln bilden, in Peploptera nicht; letztere ist aufserdem noch an dem tiefen Quereindruck des Thorax zu unterscheiden, in den die aufgebogene Kante des Mittellappens vorn abfällt, endlich mit absoluter Sicherheit am Verlaufe der neunten Pnnktreihe der Flgd., welche selbst bei den verworren punktierten Arten vorhanden ist und erst dicht an der Schulterbeule bogenförmig nach aufsen läuft, sodafs sich der letzte Zwischenstreifen über der hinteren Hälfte des Seitenlappens stark verbreitern mufs. Diese verbreiterte Stelle hat niemals primäre, böchstens feine, sekundäre Punkte, während bei Diapromorpha die primären Punkte bis an den Rand des Seitenlappens reichen.

Die Gattung Diapromorpha ist auf Afrika beschränkt (die indischen Arten sind abweichend gebildet) und enthält gegenwärtig nur 8 bis 10 Species:

1. Gruppe. Das Prosternum bildet vor den Hüften eine Beule oder einen kurzen Längskiel, ist dann sehr schmal, vertieft, unsichtbar, und endet hinten ziemiich tief in einen abfallenden, nach unten verbreiterten Fortsatz.

1. Oberlippe, Fühler und Beine rotgelb, Thorax und Fld. apfelgrün oder gelb, ersteres mit einer schwarzen, bogenförmigen Querbinde an der Basis und zwei schwarzen Makeln davor, die hinten oft mit der Binde vereint sind; Fld. nach hinten verengt, mit drei schwarzen Querbinden, von denen die erste am Aufsenrande der Schulterbeule mehr oder weniger tief winkelig ausgeschnitten ist. Länge 9-12 mill. Ganz Mittelafrika . . . .

trifasciata $\mathrm{F}$.

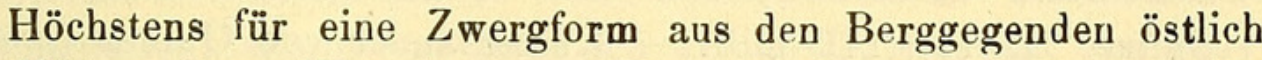
vom Kilimandjaro (Usambara und Britisch Ostafrika), 6,5-8 mill. lang, bei der die beiden Thoraxflecke oft frei und die ersten beiden Querbinden der Fld. häufig in ihre urspünglichen Makeln aufgelöst sind, halte ich jetzt Diapr. Juno Ws., Archiv 1903, I, 2, 198. Die erste Querbinde entsteht aus 4 schwarzen Makeln: eine gemeinschaftlich, vor $\frac{1}{3}$ der Länge, eine auf der Schulterbeule, eine in der 
Mitte $\mathbf{z w i s c h e n}$ den beiden vorigen, die vierte schräg nach aufsen und hinten vom Schulterflecke und oft mit diesem zu einer winkeligen Makel zusamınengeflossen. Bei den übrigen Arten mit schwarzen Querbinden entsteht die erste aus drei Makeln: eine nahe der Naht, dicht vor $\frac{1}{3}$ Länge, die zweite auf der Schulter, die dritte direkt hinter dieser; es kann sich bei ihnen daher kein winkeliger Schulterausschnitt bilden,

1'. Oberlippe, Fühler und Beine schwarz, an den Fühlern nur die Spitze des ersten Gliedes und Glied 2 und 3 rostrot. Fld. fast parallel, jede mit 5 schwarzen Makeln: 2, 2, 1. Makel 1 und 2 gestreckt, 1 weit hinter der Basis, vor der Mitte, näher der Naht als dem Seitenrande, 2 viel weiter vorgerückt, auf der Schulter, an oder nahe der Basis beginnend; die beiden folgenden hinter der Mitte, 3 weiter nach hinten gerückt als 4 am Seitenrande, Makel 5 endlich befindet sich am Hinterrande. Von diesen Makeln fliefsen gewöhnlich 3 und 4 zu einer, zuletzt gemeinschaftlichen Querbinde zusammen, die sich später durch einen Ast in der Mitte jeder Fld. mit dem Spitzenflecke vereint. Die beiden vorderen Makeln verlängern sich nach hinten und es verbindet sich häufig Makel 2 und 4, seltener auch 1 und 3. Aufserdem ist die Naht von $\frac{1}{3}$ der Länge ab schwarz gesäumt. L. $9-11,5$ mill. - Westliches Mittelafrika, östlich noch bei Bismarckburg in Deutsch Ostafrika, an der Südostecke des Tanganyika (bicruciata Illig.) . . . . . . . . . . . picta F.

2. Gruppe. Prosternum ähnlich wie bei der vorigen, aber hinten in einen aufsteigenden Fortsatz verlängert.

1. Dieser Fortsatz bildet eine lange, schräg ansteigende dreieckige Platte. Körper schwarz, oberseits glänzend, ein Saum am Vorderrande des Thorax (oft breit unterbrochen) und ein Seitensaum, sowie die Fld. hell bräunlich oder rötlich gelb, letztere fast parallel, an der Naht und am Hinterrande fein schwarz gesäumt, häufig noch mit einem schwarzen Flecke auf der Schulter, einer schwarzen Querbinde hinter der Mitte, weder Naht- noch Seitenrand berührend, und einer saumförmigen Makel in der Spitze. L. 12-14,5 mill. -- Küstenländer von Guinea (procera Jac. Transact. 1895, 165) Ann. Fr. 1891 Bull. 173 ingens Lef. 1'. Fortsatz kurz, dreieckig oder höckerförmig. . . 2.

2. Thorax wenig glänzend, von hinten und oben betrachtet nach vorn fast gleichmälsig stark verengt, konisch. Fld. einfarbig bräunlich gelb oder variabel gezeichnet, zuletzt mit 3 schwarzen oder bläulich schwarzen Querbinden, von denen die beiden letzten 
in der Mitte zusammenhängen, so dafs dann vor der Spitze je zwei gelbe, schräg liegende Makeln übrig bleiben.

a. Schienen und Tarsen rostrot. L. 9-12,5 mill. - Küstenländer von Guinea argentata $\mathrm{F}$.

b. Beine einfarbig schwarz. Grölse und Verbreitungsbezirk wie bei der vorigen und von dieser wohl nicht spezifisch verschieden

concinna Lac.

2'. Thorax stark glänzend, halbkugelig. Schwarz, Unterseite und Beine, namentlich der Bauch oft mit grün- oder bläulichem Metallschimmer, wie bei den meisten übrigen Arten. Thorax tiefschwarz, vorn und an den Seiten gelb gerandet, Fld. gelb, mit 4 tiefschwarzen Querbinden, von denen die letzte nur einen schmalen Saum am Hinterrande bildet. Die erste und zweite gelbe Querbinde sind mäfsig breit, die dritte ist schmal, zuweilen in der Mitte unterbrochen, die vierte bildet auf jeder Decke eine schmale Quermakel. L. 6,5-9 mill. - Ostafrika tetten s is Gerst.

3. Gruppe. Das Prosternum steigt vorn in schräger Richtung auf, bildet dann einen gleichhohen oder schwach konkaven deutlichen Längsstreifen und endet in einen überhängenden Fortsatz

1. Hinterrand der Fld. schwarz gesäumt. . . . . 2.

1'. Der Hinterrand der Fld. wird von einer roten oder rötlichgelben Quermakel eingenommen. Fühler, Schienen und Tarsen hell rostrot. Fld. aufser der roten Spitze gelb, mit 3 gemeinschaftlichen schwarzen Querbinden, von denen die erste aufsen abgekürzt ist und vor der Schulter die Basis nicht erreicht. Lg. 8-11 mill. Ostafrika (I). tigrina Jac., Transact. 1901, 220, t. 10, fig. 8) . .

trizonata Fairm.

2. Schienen und Tarsen rostrot. Fld. dicht punktiert, an der Spitze durch feine Risse und Runzeln matt, die erste schwarze Querbinde verlängert sich vor der Schulter bis zur Pasis, die dritte (rote, seltener gelbe) Querbinde häufig in zwei gerundete Makeln aufgelöst. L. 9,5-13,5 mill. - Ostafrika (Wiegm. Arch. 1871, I, 80)

haemorihagica Gerst.

Diese Art dürfte mit D. Walleri Baly, Transact. 1864, 224, zusammenfallen, wenigstens finde ich keinen Unterschied aus Baly's Beschreibung heraus. Diese müfste dann nach den nicht häufigen Stücken entworfen sein, bei denen die beiden Makeln an der Spitze der Fld. nicht rot, wie in haemorrhagica, sondern hellgelb gefärbt sind.

2'. Beine einfarbig schwarz. Fld. wenig dicht punktiert, auch an der Spitze glänzend; die erste schwarze Querbinde erreicht vor 
der Schulterbeule nicht die Basis, die beiden gelben Makeln, die von der dritten hellen Querbinde übrig geblieben sind, kleiner wie in der vorigen, nicht rund, sondern eckig, und eine Spur eingeschnürt. Lg. 11-12 mill. - Britisch Central-Afrika

Scelodonta albidovittata Baly. zebra Lac.

Syagrus Bodongi: Oblongo-ovalis, niger, nitidus, labro antennisque basi rufescentibus; capite crebre subruguloso-punctato, vertice utrinque longitudinaliter rugoso, prolhorace alutaceo, dense punctato, elytris punctato-striatis, intervallis laevibus, convexiusculis, humeris prominulis. - Long. 6 mill.

Dem S. morio Harold ${ }^{1}$ ) nahestehend, aber in folgenden Stücken abweichend: Der Körper ist kürzer gebaut, die Stirn zwischen den Augen breiter, dicht und etwas runzelig-, der Scheitel längsstreifig punktiert, mit langen, leistenförmigen $\mathrm{Z}$ wischenstreifen; der Thorax ist dichter gewirkt, an den Seiten vor der Mitte mehr gerundet, die Fld. treten an der Basis stärker heraus und sind in den Schultern am breitesten, dann bis zur Mitte unbedeutend verengt, dahinter stärker verschmälert, mit kräftig punktierten Streifen und glatten, gewölbten Zwischenstreifen. Bei $S$. morio sind die Fld. nicht in den Schultern, sondern vor der Mitte am breitesten, die Schulterbeule ist kleiner und tritt weniger nach aufsen, die $\mathbf{Z w}_{w}$ schenstreifen der Punktreihen sind äufserst fein und wenig dicht runzelig-punktiert und, wenigstens im mittleren Teile, völlig eben.

Meniellus Kohlschütteri Ws. Archiv f. Naturg. 1903, I, 2, 203.

Plagiodera opacicollis Ws. 1. c. 205. Bei Umtali häufig. Manche Exemplare haben auf dem Thorax einen etwas stärkeren Glanz, sie sind matt seidenschimmernd; bei andern ist der Körperumrifs weniger gerundet, fast regelmäfsig elliptisch, die Fld. sind lebhaft und gesättigt metallisch-grün, zuweilen mit goldigem Schimmer.

Chrysomela Simonsi Baly 1878. Die Stücke von Umtali, nahe der Gegend, von der Baly seine Art beschrieb, = confluens Gerst. 1855.

Bacteriaspis scutellata Baly, Ent. Monthl. Mag. XIV, 205 (Februar 1878). Dieses Tier gehört nicht zu Prosmidia, wie ich

1) Mit dieser Art ist Syagrus puncticollis Lef., Ann. Fr. 1877, 320, identisch; Harold beschrieb dieselbe von Natal (die Type befindet sich im hiesigen $\mathrm{kgl}$. Museum), Lefèvre von Zanzibar. Die Art ist von Britisch Ostafrika bis Natal verbreitet und sehr häufig. 
D. E. Z. 1905, 45 vermutete, sondern in die Gruppe der Phyllobroticinen. Baly betrachtete dasselbe als eine Aulacophora, womit es nur eine entfernte Aehnlichkeit, aber keine Verwandtschaft hat. Die Epipleuren der Fld. scheinen ganz zu fehlen, in Wirklichkeit aber liegen sie sehr tief, beginnen ganz schmal ein Stück hinter der Schulter, erweitern sich dann allmählich bis zur Mitte, verengen sich ähnlich dahinter und endigen nahe der Spitze. Der Thorax hat keine durchlaufende Qnerfurche, sondern nur eine Querrinne jederseits, die Klauen sind nicht gespalten, sondern an der Basis gezähnt; sie haben hier ein grofses, zahnförmiges Anhängsel, welches durch einen mäfsig langen, schrägen Einschnitt von dem oberen Teile getrennt wird. Vorder- und Mittelhüften stofsen zusammen, die vorderen Gelenkhöhlen sind offen, die Hinterschienen haben einen kurzen Enddorn. Sehr bemerkenswert ist der Bau des Thorax, des Schildchens und der Flügeldeckenbasis beim $\widehat{\jmath}$ : durch einen leichten Quereindruck an der Basis des Thorax jederseits wird der kleine, nach hinten wenig verlängerte Mittelzipfel desselben höckerartig emporgehoben. Der höchste Teil der Beule trägt ein Grübchen. Das Schildchen steht aufrecbt, neigt sich mit der dicken, oben abgeflachten Spitze etwas nach hinten und sendet dann, vom Hinterrande aus, einen fast häutig erscheinenden, zungenförmigen, oben muldenartig vertieften Fortsatz aus, der sich nach vorn biegt und wenig über den Thoraxhöcker hinaus ragt. Die Basis der Fld. ist in der inneren Hälfte quer grubenförmig vertieft. Diese Vertiefung wird aufsen durch eine Beule des Basalrandes begrenzt und innen von der entsprechenden Grube der andern Decke durch die hochliegende Nahtkante geschieden, die vorn verbreitert und mit einem Grübchen besetzt ist. Aufserdem ist beim $\widetilde{\sigma}$ der vierte Bauchring in der Mitte verengt, der fünfte so lang wie die vorhergehenden zusammen, mit einer tiefen, ganzen Längsgrube, die vorn schmal, hinten breit ist und hier den gesamten Mittelzipfel einnimmt.

Hyperacantha mililaris Jac. Transact. $1903,19=$ seminigra All. 1889.

Asbecesta cyanipennis Har.

Asbecesta capensis All. Ann. Fr. 1888, 326. Bei Umtali gemein. Die Art ist blafs rötlich gelbbraun, die Fld. heller, weifslich gelb, Fühler schwarz, das erste Glied gänzlich, oder die ersten 3-4 Glieder unterseits rötlich gelbbraun, eine kleine Makel auf dem Scheitel, eine gröfsere dahinter auf dem Thorax vor der Querfurche, Schildchen, Knie, Spitze der Schienen und die Tarsen schwarz; 
Fld. dicht punktiert, gleichmäfsig schwach gewölbt, ohne Längseindruck. Die Stirn ist fast glatt, oder auf einem gröfseren Raume jederseits neben dem Auge kräftig und dicht runzelig-punktiert.

Die dunkelsten Stücke, die ich als ab. cincıa bezeichne, haben öfter den ganzen Scheitel schwarz, auf dem Thorax vor der Querfurche drei grofse schwarze Makeln, von denen die mittelste zuweilen strichförmig bis an das Schildchen verlängert ist, sowie die Naht und ein Seitensaum der Fld. schwarz. Letzterer ist am Hinterrande oft erweitert und bedeckt vorn manchmal den grölsten Teil der Schulterbeule.

Asbecesta pectoralis Jac. Transact. 1895, 327. Die helle Färbung der Fühlerwurzel ist variabel; gewöhnlich sind, wie Jacoby angibt, nur die ersten drei Glieder rötlich gelbbraun, öfter jedoch, namentlich beim $ᄋ$, die vier ersten Glieder ganz, der gröfste Teil des fünften und die Basis des sechsten Gliedes. Die Fld. sind vorherrschend metallisch dunkelgrün.

Asbecesta melanocephala Jac. 1. c. 327. Die Fld. sind häufig dunkelblau, mit violettem Anfluge.

Asbecesta sobrina: Nigra, antennis basi ferrugineis, coxis tibiarumque apice interdum rufo-piceis, prothorace parce punctulato, elytris coeruleo-nigris, crebre punctatis, pone humeros longiludinaliter impressis. - Long. 3,5-4 mill.

Den beiden vorigen in Gröfse und Körperform ähnlich, aber auf den Fld. dunkler gefärbt und weniger stark, besonders flacher punktiert, der Thorax deutlich breiter gebant als in melunocephala. An den Füblern sind die ersten vier Glieder rostrot, das erste Glied stets, das vierte öfter oberseits schwärzlich, die Hüften und die Spitze der Schienen beim 오 zuweilen rötlich pechbraun, die übrigen Körperteile schwarz, glänzend, die Fld. bläulich schwarz, dicht punktiert, weniger glänzend. Auf jeder Decke läuft vom Innenrande der Schulterbeule bis hinter die Mitte eine Längsvertiefung, welche aulsen eine schwache, stumpfe Längskante emporhebt, ähnlich wie bei pecloralis und melanocephala. Das $\sigma^{\top}$ hat vom Hinterrande des letzten Bauchringes aus jederseits einen Einschnitt, der bedeutend schräger nach innen und vorn läuft wie in pectoralis.

Asbecesta usambarica Ws. D. E. Z. 1901, 306. Einige Stücke, den gröfsten von Usambara ähnlich, haben die sçhwarze Basalbinde der Fld. ziemlich breit. 
Cynortina modesta Jac. Transact. 1895, 339. Mit Recht stellte Jacoby dies Tier nur fraglich zu Cynorla, denn es weicht meiner Meinung nach spezifisch in folgenden Punkten ab: die Fühler sind verbältnismälsig kräftig gebaut, Glied 2 ist ziemlich kurz, 3 etwas länger, an der Spitze schief abgeschnitten, 4 wieder etwas länger als 3 und den folgenden ungefähr gleich, 5 und 6 von diesen ebenfalls an der Spitze schief abgestutzt. Thorax ohne Eindrücke. Fld. gleichmäfsig und fein verworren punktiert, ihre Epipleuren nahe der Mitte schon sehr schmal, weit vor der Spitze endigend. Metatarsus nur mälsig gestreckt, nicht ganz so lang wie Glied 2 und 3 zusammen. Das $\sigma^{\wedge}$ ron Cynortina bat am Hinterrande des letzten Bauchringes zwei kurze, schiefe Einschnitte, durch die ein quer viereckiger, nach hinten erweiterter Mittelzipfel entsteht, in den die breite und tiefe, vorn abgekürzte Mittelrinne hineinläuft.

Megalognatha Bodongi: Atra, ventre plus minusve fulvo, prolhorace pone apicem profunde lransversim impresso, parte apicali cristam elevalam angulatam formante, elytris creberrime subrugulosopunctatis. - Long. 6-7 mill.

Mas: Antennarum articulis quinto et sexto sat brevibus, interne deplanatis, septimo angulatim dilatato, hamato, caeteris interne canaliculatis, prothorace nitidulo, sat crebre punclalo.

Femina: Antennis subfiliformibus, prothorace in disco rugosopunctato.

Der M. apicalis Ws. Archiv f. Naturg. 1904, I, 2, 169, sehr ähnlich, aber die Fld. einfarbig schwarz, noch dichter punktiert, die Fübler des ð ganz abweichend gebaut. Tiefschwarz und ziemlich matt, der vertiefte Querstreifen des Kopfschildes, der Vorderrand der Oberlippe und die Taster rötlich, der Bauch rotgelb ( $\left.\sigma^{\top}\right)$ oder dunkler, vorn oft schwärzlich (q), an den Fühlern sind die ersten sieben Glieder wenig dicht behaart, eine Spur glänzend, die vier Endglieder matt. Glied 1 lang, keulenförmig, 2 kurz und dünn; beim $\sigma^{\star}$ sind die drei folgenden Glieder allmählich verbreitert, unten flach gedrückt, am Innenrande leicht und schmal vertieft, Glied 3 nicht ganz so lang als 1, 4 kürzer, wenig länger als an der Spitze breit, 5 kürzer als 4 , etwas breiter wie lang, 6 noch kürzer und fast nur halb so breit als das vorhergehende, 7 das breiteste, stark nach aufsen erweitert, diese Erweiterunng an der Basis im Bogen ausgerandet, die vier Endglieder etwas zusammengedrückt und am Aufsenrande mit einer breiten und tiefen Längs- 
rinne versehen. Beim $q$ ist Glied 3 so lang als 1,4 und die folgenden sind wenig an Länge verschieden, jedes kürzer als 3, mit dünner Basis und breiterer Spitze. Der Vorderrand des Thorax steigt zu einem niedrigen, dicht und sehr fein punktierten $\left(\sigma^{\top}\right)$, oder hohen und fast glatten Querstreifen auf, welcher in der Mitte am breitesten, jederseits davon verschmälert, hinten also stumpfwinkelig geformt und beim $q$ scharfrandig und leicht überhängend ist. Er fällt nach hinten in eine weite Quervertiefung ab, die aus vier Gruben besteht, einer grofsen aufsen und einer kleineren innen neben der kielförmigen Mittellinie. Diese Gruben sind beim $\sigma^{\top}$ sparsam punktiert und besser ausgeprägt wie die des 우, bei dem sie dicht und etwas runzelig punktiert sind. Der Raum hinter dem Quereindrucke ist mälsig dicht punktiert $\left({ }^{\top}\right)$, oder dicht runzelig punktiert (ㅇ).

Hemixantha pallida Jac. Proceed. 1897, 576. Stücke von Umtali werden bis 8,5 mill. lang; ein Exemplar in der Sammlung Ertl, von Iringa, mifst nur 7 mill.

\section{Eutornus Clark.}

Nach dem im allgemeinen spärlich einlaufenden HalticinenMateriale lälst sich heute nur vermuten, dafs meine Gattung Philopona. Archiv f. Naturg. 1903, I, 2, 216, mit Eutornus nahe verwandt und später rielleicht als Abteilung zu rerbinden sein dürfte. Die typische Art dieser Gattung, E. africanus Clark, ist über das ganze südliche Dreieck Afrikas verbreitet; ich habe nun auch Stücke von Marienberg bei Bukoba am Ukerewe und von Umtali (Bodong) gesehen, welche nicht nur jederseits eine schwärzliche Makel auf dem Thorax besitzen, wie E. rugicollis Jac. (Oedionychis), Proceed. 1899,342 , t. 21 , fig. 1, sondern dazwischen noch einen, oder drei $(2,1)$ ähnlich gefärbte kleinere Flecke. Es unterliegt wohl keinem $\mathrm{Z}$ weifel, dafs rugicollis Jac. von africanus Clark nicht spezifisch zu trennen ist. Diese Art ist durch die kräftige, zuweilen runzelige Punktierung des Thorax (welche stärker als die der Fld. ist) und die schwarze oder schwarzgrüne ziemlich breite, hinten abgekürzte Längsbinde jeder Fld. sehr kenntlich. Weder Clark noch Jacoby erwähnen den sehr auffälligen Längseindruck, welcher die Längsbinde an der Aufsenseite begrenzt, an der Basis beginnt, den Schulterhöcker absetz.t und mehr oder weniger weit dahinter verlängert ist. Die Philopona-Arten sind glänzender, oberseits feiner und sparsamer punktiert, haben ein schlankeres, zugespitztes Endglied der Maxillartaster, einen breit abgesetzten, 
flachen Seitenrand des Thorax und einen Borstenkegel in den Hinterecken desselben. Da Jacoby in den Proceed. 1900 p. 266 seine Oedionychis rugicollis und africana Jac. $1894=$ Clarki Jac. 1900 = vernicata Gerst. 1871 als Eutornus ansieht, verbindet er schon völlig mit dieser Gattung die Philopona Ws, womit ich mich augenblicklich nicht einverstanden erklären kann.

Philopona rufinasus: Oblongo-ovalis, testaceo-flava, nitida, elytris saepe stramineis, femorum apice, pectore, ventris basi, antennarum articulis septem ultimis, capite (clypeo excepto), punctis duobus prothoracis, scutello, sutura maculisque tribus elytrorum nigris, prothorace ante basin impressione transversa evidenter punctata, elytris pnnctulatis pone basin punctatis. Long. 4,5-5 mill. Umtali.

Der Ph. vernicata Gerst. sehr äbnlich, aber gröfser, namentlich breiter gebaut und durch folgende Punkte rerschieden: Das Kopfschild ist rötlich gelb, wenig dicht und sehr fein punktuliert, oben durch eine deutliche bogenförmige Punktreihe von den etwas niedriger liegenden Stirnböckerchen abgesetzt; die Taster sind hell gefärbt, die Stirn in der Mitte glatt, auf einem Streifen neben den Augen ziemlich dicht, nach oben zu sehr dicht und runzelig-punktiert. Der Thorax hat vor der Basis einen tieferen, in der Mitte leicht unterbrochenen, dichter und stärker punktierten Quereindruck, der Vorderrand ist angedunkelt, dahinter liegt jederseits nahe der Mittellinie eine kleine, schwarze Makel. Auf den Fld. ist der Längseindruck an der Innenseite der Schulterbeule sehr deutlich, lang, kräftig punktiert, und diese Punktierung setzt sich auf einem leicht vertieften Querstreifen hinter der wenig aufgetriebenen Basis bis an die Naht fort, wird aber nach innen zu etwas feiner; die übrigen Teile sind fein und verloschen punktuliert, nur im ersten Viertel liegt neben dem Schildchen und der Naht ein Punktstreifen. Die drei schwarzen Makeln jeder Fld. haben ungefähr dieselbe Lage wie in vernicala und sind in Form und Gröfse veränderlich; die erste bedeckt die Schulter, die zweite ist langgestreckt, bald einfach, strichförmig, bald winkelig, die dritte in der Spitze ist klein. Bei einem Stücke dehnt sich Makel 2 sehr aus und hängt breit mit dem Nahtsaum zusammen.

Plyysonychis violaceipennis Baly Ent. Monthl. Mag. 14, 204 (Febr. 1878). Der Autor bezeichnete diese Spezies als eine Physoma, sie steht jedoch der Physonychis subopaca m., Archiv f. Naturg. 1902, I, 2, 169, aus Usambara so nahe, dals die spezifische 
334 J. Weise: Ueber Chrysomeliden und Coccinelliden aus Rhodesia.

Verschiedenheit beider erst noch weiter geprüft werden mufs. Ph. subopaca hat mälsig lange Fubler, an denen die vier Endglieder schwärzlich sind; jedes von diesen ist nicht ganz doppelt so lang als breit; die Vorderecken des Thorax haben eine breit abgerundete Spitze und die Fld. sind äufserst dicht punktiert und sehen auf der Scheibe gleichmäfsig bläulich kupferrot aus, weil die im Grunde glänzend schwarz gefärbten Punkte die Farbe der schmalen Zwischenräume nicht beeinträchtigen. Die Stücke der violaceipennis aus Umtali haben bedeutend längere und auch dunklere Fühler, an denen in der Regel nur die vier ersten Glieder rötlich, die vier letzten fast dreifach länger als breit sind; ebenso treten die Vorderecken des Thorax spitz zahnförmig nach vorn und aufsen und die Fld. sind in den schmalen Zwischenräumen lebhafter kupferig gefärbt, die Punkte im Innern glänzend metallisch grün. Es entsteht so keine einheitliche, sondern eine zwischen grün und kupferrot wechselnde Farbe der Scheibe.

Metriopepla lividula Fairm., Ann. Belg. 1882, 57, wurde bereits ron Waterhouse, Cist. II, 229, 17. Mai 1877, als Epistictia inornata beschrieben; sie mufs also Metriopepla inornata Waterh. heifsen.

Aspidomorpha 4-maculata Oliv. aber. signaticollis m. Unterseite häufig schwarz, wie bei der nahe den grofsen Seen vorkommenden Form, nur die Mitte der Vorder- und Mittelbrust, eine zweilappige Makel an der Basis der Hinterbrust, sowie die Mitte und die Seiten des Bauches gelb. Die drei schwarzen Thoraxpunkte haben sich ausgedehnt, der mittlere bildet eine dreieckige, nach vorn verbreiterte Makel, zuletzt einen grofsen und breiten, nach vorn geöffneten Bogen, der von einem Rande der Scheibe bis zum andern reicht. Der Seitenpunkt bat sich zu einem rechtwinkeligen Flecke vergröfsert, dessen hinterer, querliegender Schenkel innen bis nahe an das Schildchen zieht; der äufsere, nach vorn gerichtete Schenkel ist kürzer und dicker. Diese Form erhielt ich auch aus Uhehe durch Hrn. Prof. Hauser.

Aspidomorpha Severini Spaeth, Ann. Belg. 1902, 447.

Solanophila Bodongi: Subhemisphaerica, subtus nigra, supra teslacea, dense subliliterque ochraceo-pubescens, elytris creberrime punctulatis, singulo maculis sex nigris, 2, 2, 2 oblique digestis. Long. 5 mill.

Var. a: Subtus cum pedibus testacea. 
In der Zeichnung etwas an Ep. Dufouri Muls. erinnernd, aber ziemlich so breit als lang, gerundet, gewölbt. Thorax fast dreimal so breit als lang, am Hinterrande ziemlich gleichmäfsig, an den Seiten stark gerundet, am Vorderrande tief ausgeschnitten, so dafs die Vorderecken weit vorragen. Fld. äufserst dicht und fein punktiert und dicht gelblich behaart, ohne bemerkbare gröfsere Punkte, jede mit 6 ziemlich grofsen, schwarzen, gerundeten, schwarz behaarten Makeln, die in drei schiefen Querreihen, annähernd ähnlich wie bei Ep. chrysomelina angeordnet sind. Makel 1 befindet sich an der Basis nahe dem Schildchen, 2 weiter von der Basis entfernt auf der Schulter, 3, unmittelbar vor der Mitte, ist gewöhnlich breiter als lang, die gröfste von allen, und ungefähr um ihren Querdurchmesser von der Naht abstehend, 4, weiter nach hinten gerückt, liegt in der Mitte zwischen der dritten Makel und dem Aufsenrande, 5, dicht an der Naht etwas hinter der Mitte, ist die kleinste, zuweilen schräg nach vorn und aufsen mit der dritten verbunden, 6 ist von der Naht und dem Hinterrande gleichweit, von der hinteren Nahtecke nur wenig weiter entfernt. $\mathrm{Zu}$ beachten ist die Lage der dritten Makel, von Makel 1 und der Naht rerhältnismäfsig weit abgerückt und der Makel 5 genähert. Die Bauchlinien sind vollstandig und reichen bis an das letzte Viertel des ersten Ringes.

Solanophila 14-signata Rche.Voy. Galin. Abyss. III, 1847, 418.

Halyzia exigueguttata Gorh. Ann. Mag. 1901, $407=$ pardalina Gerst. (sub Chilomenes) 1871.

Alesia inclusa Muls. Bei allen Stücken von Umtali ist der äufsere Bogen der schwarzen Längsbinde vor der Mitte jeder Fld̀. vollständig, so dafs dort eine grofse, runde, gelbe Makel völlig eingeschlossen wird.

Ortalia punctata Ws. D. E. Z. 1888, p. 89, Var.? Es wurden bisher bei Umtali nur 2 Exemplare gefangen, die sich von der genannten Art durch einfarbig schwarze Mittel- und Hinterbrust und durch einen überzäbligen gelben Fleck in der Schulterecke der Fld. unterscheiden. Die Zeichnung der Fld. erinnert daher an die von O. 12-maculata Ws., weil auf jeder Decke sechs gelbe Flecke vorhanden sind: 2, 2, 1. Die Beine sind wie in punctata schwarz, nur die Tarsen rötlich.

Aulis Gorhami Ws. Archiv f. Naturg. 1904, I, 1, 61. 


\section{$2 \mathrm{BHL}$ Biodiversity Heritage Library}

Weise, Julius. 1905. "Ueber Chrysomeliden und Coccinelliden aus Rhodesia." Deutsche entomologische Zeitschrift 1905, 321-335.

View This Item Online: https://www.biodiversitylibrary.org/item/103316

Permalink: https://www.biodiversitylibrary.org/partpdf/82812

\section{Holding Institution}

Harvard University, Museum of Comparative Zoology, Ernst Mayr Library

\section{Sponsored by}

Harvard University, Museum of Comparative Zoology, Ernst Mayr Library

\section{Copyright \& Reuse}

Copyright Status: Public domain. The BHL considers that this work is no longer under copyright protection.

This document was created from content at the Biodiversity Heritage Library, the world's largest open access digital library for biodiversity literature and archives. Visit BHL at https://www.biodiversitylibrary.org. 